\title{
Some remarks on finite submodules of the unramified Iwasawa module of totally real fields
}

\author{
Dedicated to Professor Keiichi Komatsu on the Occasion of his 70th birthday
}

\author{
By Satoshi FujII
}

Faculty of Education, Shimane University, 1060 Nishikawatsucho, Matsue, Shimane 690-8504, Japan

(Communicated by Shigefumi MorI, M.J.A., Oct. 12, 2020)

\begin{abstract}
Let $k$ be a number field and $p$ a prime number. It is conjectured by Greenberg that the Iwasawa $\lambda$ - and $\mu$-invariants of the cyclotomic $\mathbf{Z}_{p}$-extension of $k$ always vanish if $k$ is totally real. In this article, we will discuss a weak version of Greenberg's conjecture, and give results analogous to Greenberg's and Ozaki's results.
\end{abstract}

Key words: Unramified Iwasawa modules; finite submodules.

1. Introduction. Let $p$ be a prime number and $k / \mathbf{Q}$ a finite extension, where denote by $\mathbf{Q}$ the field of rational numbers. Let $\mathbf{Z}_{p}$ be the ring of $p$-adic integers. An algebraic extension $K / k$ is called a $\mathbf{Z}_{p}$-extension if $K / k$ is a Galois extension and $\operatorname{Gal}(K / k) \simeq \mathbf{Z}_{p}$ as topological groups. Let $K / k$ be a $\mathbf{Z}_{p}$-extension. For each non-negative integer $n$, let $k_{n}$ denote the unique intermediate field of $K / k$ such that $\left[k_{n}: k\right]=p^{n}$. For a number field $F$, let $A_{F}$ be the $p$-part of the ideal class group of $F$. Then, it is shown by Iwasawa that there exist non-negative integers $\lambda, \mu$ and an integer $\nu$ depending only on $K / k$ such that the normalized additive $p$-adic valuation of the order of $A_{k_{n}}$ is described as $\lambda n+$ $\mu p^{n}+\nu$ for all sufficiently large $n$. It is conjectured by Greenberg [1] that $\lambda$ and $\mu$ always vanish for the cyclotomic $\mathbf{Z}_{p}$-extension $k_{\infty} / k$ of each totally real field $k$ (for short we say GC). In the same paper [1], Greenberg showed the following

Theorem (Greenberg [1]). Let $k$ be a totally real field such that $k_{\infty}$ has the unique prime lying above $p$ and that $k_{\infty} / k$ is totally ramified at the ramified prime. Then the following two conditions are equivalent.

(a) $\lambda=\mu=0$.

(b) The lifting mapping $A_{k} \rightarrow A_{k_{n}}$ is trivial for some $n \geq 1$.

For a $\mathbf{Z}_{p}$-extension $K / k$, let $X_{K}$ be the Galois group of the maximal unramified abelian pro- $p$ extension $L_{K} / K$, which is called the unramified Iwasawa module of $K / k$. The module $X_{K}$ is also

2020 Mathematics Subject Classification. Primary 11R23. defined to be the projective $\operatorname{limit} \underset{n}{\lim } A_{k_{n}}$ with respect to the norm maps. Then the complete group ring $\mathbf{Z}_{p}[[\operatorname{Gal}(K / k)]]$ with coefficients in $\mathbf{Z}_{p}$ acts on $X_{K}$. Let $X_{K}^{0}$ be the maximal finite $\mathbf{Z}_{p}[[\operatorname{Gal}(K / k)]]$-submodule of $X_{K}$. Then $\lambda=\mu=0$ if and only if $X_{K}=X_{K}^{0}$. From this point of view, we can consider a weak version of Greenberg's conjecture as follows: When $k$ is a totally real field, if $X_{k_{\infty}} \neq 0$, then does it hold that $X_{k_{\infty}}^{0} \neq 0$ ? (for short we say WGC). For a prime number $p$ and a totally real field $k$, if GC holds for $p$ and $k$ then WGC holds for $p$ and $k$. The author thinks that the study of WGC is important to study GC. Ozaki [3] obtained the following result concerning WGC. We shall introduce a slightly modified statement.

Theorem (Ozaki [3]). Let $p$ be an odd prime number and $k$ a totally real field in which $p$ splits completely. Suppose that Leopoldt's conjecture for $p$ and $k$ holds. Then the following two conditions are equivalent.

(a) $X_{k_{\infty}}^{0} \neq 0$.

(b) $L_{k_{\infty}} \neq M_{k_{\infty}}$.

Here, $M_{k_{\infty}} / k_{\infty}$ stands for the maximal abelian pro-p extension unramified outside all primes lying above $p$.

In this article, we will study WGC and give analogous results to Greenberg's and Ozaki's theorems. From here to the end of this article, we assume the following

Assumption (A). Let $p$ be a prime number and $k$ a totally real field such that $k_{\infty}$ has the unique 
prime lying above $p$ and that $k_{\infty} / k$ is totally ramified at the ramified prime.

The first result is a WGC analogue of Greenberg's theorem.

Theorem 1. Under the assumption $(A)$, the following two conditions are equivalent.

(a) $X_{k_{\infty}}^{0} \neq 0$.

(b) The lifting mapping $A_{k} \rightarrow A_{k_{n}}$ is not injective for some $n \geq 1$.

The second result is analogous to Ozaki's result when $p$ does not split in $k / \mathbf{Q}$.

Theorem 2. Suppose that the assumption (A) is satisfied. Let $U^{1}$ be the local principal unit group of $k$ with respect to the unique prime $\mathfrak{p}$ lying above $p$, and $E^{1}$ the completion the group of units of $k$ congruent to 1 modulo $\mathfrak{p}$ in $U^{1}$. Suppose that Leopoldt's conjecture for $p$ and $k$ holds, and that $U^{1} / E^{1}$ is free over $\mathbf{Z}_{p}$. Then the following two conditions are equivalent.

(a) $X_{k_{\infty}}^{0} \neq 0$

(b) $L_{k_{\infty}} \neq M_{k_{\infty}}$.

We must mention here that, on Theorem 2, the oddness of $p$ is not needed.

We shall set some notations of this article. For a $\mathbf{Z}_{p}$-module $A$, let $\operatorname{Tor}_{\mathbf{Z}_{p}} A$ be the submodule of $A$ which consists of all $\mathbf{Z}_{p}$-torsion elements of $A$. For a topological group $G$ and a topological $G$-module $M$, let $M^{G}$ and $M_{G}$ be the maximal submodule and the maximal quotient module of $M$ on which $G$ acts trivially.

2. Preliminaries. We need the following lemmas.

Lemma 3 (Ozaki [2]). Let $K / k$ be a $\mathbf{Z}_{p}$-extension which is totally ramified at all ramified primes. Then we have $\operatorname{Ker}\left(A_{k_{n}} \rightarrow \underset{m}{\lim } A_{k_{m}}\right)=$ $\operatorname{Im}\left(X_{K}^{0} \rightarrow A_{k_{n}}\right)$.

Lemma 4 (See Lemma 13.15 of [4]). Let $k$ be a number field and $K / k$ a $\mathbf{Z}_{p}$-extension such that $K / k$ is ramified at only one prime $\mathfrak{p}$ and is totally ramified at $\mathfrak{p}$. Then $\left(X_{K}\right)_{\mathrm{Gal}(K / k)} \simeq A_{k}$.

3. Proof of Theorem 1. Let $\Gamma=$ $\operatorname{Gal}\left(k_{\infty} / k\right), X=X_{k_{\infty}}$ and $X^{0}=X_{k_{\infty}}^{0}$ for simplicity. By lemma 4 , we have $X_{\Gamma} \simeq A_{k}$, and hence $\left(X / X^{0}\right)_{\Gamma}$ is finite. It follows from the exact sequence

$$
0 \rightarrow\left(X / X^{0}\right)^{\Gamma} \rightarrow X / X^{0} \rightarrow X / X^{0} \rightarrow\left(X / X^{0}\right)_{\Gamma} \rightarrow 0
$$

that the characteristic ideal of $\left(X / X^{0}\right)^{\Gamma}$ is trivial. Thus, since $X / X^{0}$ has no non-trivial finite submodule, it holds that $\left(X / X^{0}\right)^{\Gamma}=0$. Hence we have the following exact sequence and commutative diagram

$$
\begin{aligned}
& 0 \rightarrow \underset{\Gamma}{X_{\Gamma}^{0}} \rightarrow \begin{array}{r}
X_{\Gamma} \\
\uparrow
\end{array} \\
& X^{0} \rightarrow A_{k}
\end{aligned}
$$

By Nakayama's lemma, we find that $X^{0} \neq 0$ if and only if $X_{\Gamma}^{0} \neq 0$. By Lemma 3 and the above exact sequence, $X^{0} \neq 0$ if and only if $A_{k} \rightarrow A_{k_{n}}$ is not injective for some $n \geq 1$.

Greenberg's theorem is also shown from the above arguments. In fact, we know that $\lambda=\mu=0$ if and only if $X^{0}=X$. By Nakayama's lemma, $X^{0}=$ $X$ if and only if $X_{\Gamma}^{0}=X_{\Gamma} \simeq A_{k}$. This assertion is equivalent to that $A_{k} \rightarrow A_{k_{n}}$ is trivial for some $n \geq$ 1 by Lemma 3 .

4. Proof of Theorem 2. For a number field $F$, let $M_{F} / F$ be the maximal abelian pro- $p$ extension unramified outside all primes lying above $p$ and $\mathfrak{X}_{F}$ its Galois group. Since Leopoldt's conjecture holds for $p$ and $k, k_{\infty}$ is the unique $\mathbf{Z}_{p}$-extension of $k$. Thus, it holds that $\mathfrak{X}_{k_{\infty}}^{\Gamma}=0$ and that $\left(\mathfrak{X}_{k_{\infty}}\right)_{\Gamma}$ is finite. Then it also holds that $\left(\mathfrak{X}_{k_{\infty}}\right)_{\Gamma} \simeq \operatorname{Tor}_{\mathbf{Z}_{p}} \mathfrak{X}_{k}$ since $\operatorname{Gal}\left(k_{\infty} / k\right)$ is $\mathbf{Z}_{p}$-free. By class field theory, we have an exact sequence $0 \rightarrow U^{1} / E^{1} \rightarrow \mathfrak{X}_{k} \rightarrow$ $A_{k} \rightarrow 0$. Similarly, there is a natural surjective map $\mathfrak{X}_{k_{\infty}} \rightarrow X$ of $\mathbf{Z}_{p}\left[\left[\operatorname{Gal}\left(k_{\infty} / k\right)\right]\right]$-modules. Since there exist surjective and isomorphic mappings

$$
\left(\mathfrak{X}_{k_{\infty}}\right)_{\Gamma} \rightarrow X_{\Gamma} \simeq A_{k},
$$

and since $\left(\mathfrak{X}_{k_{\infty}}\right)_{\Gamma}=\operatorname{Tor}_{\mathbf{Z}_{p}} \mathfrak{X}_{k}$, we have the following exact sequence

$$
0 \rightarrow \operatorname{Tor}_{\mathbf{Z}_{p}}\left(U^{1} / E^{1}\right) \rightarrow\left(\mathfrak{X}_{k_{\infty}}\right)_{\Gamma} \rightarrow A_{k} \rightarrow 0
$$

of finite abelian groups. By our assumption, it holds that $\operatorname{Tor}_{\mathbf{Z}_{p}}\left(U^{1} / E^{1}\right)=0$, and hence $\left(\mathfrak{X}_{k_{\infty}}\right)_{\Gamma} \simeq X_{\Gamma} \simeq$ $A_{k}$. Put $I=\operatorname{Gal}\left(M_{k_{\infty}} / L_{k_{\infty}}\right)$. Then we have the following exact sequence

$$
0 \rightarrow I \rightarrow \mathfrak{X}_{\infty} \rightarrow X \rightarrow 0
$$

of $\mathbf{Z}_{p}\left[\left[\operatorname{Gal}\left(k_{\infty}\right) / k\right]\right]$-modules. By taking the invariant and the co-invariant sequence, we have an exact sequence

$$
\mathfrak{X}_{k_{\infty}}^{\Gamma} \rightarrow X^{\Gamma} \rightarrow I_{\Gamma} \rightarrow\left(\mathfrak{X}_{k_{\infty}}\right)_{\Gamma} \rightarrow X_{\Gamma} \rightarrow 0 .
$$

Since we have checked that $\mathfrak{X}_{k_{\infty}}^{\Gamma}=0$ and $\left(\mathfrak{X}_{k_{\infty}}\right)_{\Gamma} \simeq$ $X_{\Gamma}$, and since $X^{\Gamma}$ is finite, it holds that $X^{\Gamma} \simeq I_{\Gamma}$ and $X^{\Gamma}=\left(X^{0}\right)^{\Gamma}$. This shows that $X^{0} \neq 0$ if and only if $I \neq 0$ by a property of finite $p$-groups and by Nakayama's lemma. This completes the proof of Theorem 2 . 
Acknowledgments. The author would like to express his thanks to Tsuyoshi Itoh, Yasushi Mizusawa and Takayuki Morisawa for giving him an opportunity to talk on contexts of this article for the celebration seminar of Prof. Keiichi Komatsu's 70th birthday. The research of this article was partly supported by JSPS KAKENHI Grant numbers JP15K04791, JP18K03259.

\section{References}

[ 1 ] R. Greenberg, On the Iwasawa invariants of totally real number fields, Amer. J. Math. 98 (1976), no. 1, 263-284.

[ 2 ] M. Ozaki, A note on the capitulation in $\mathbf{Z}_{p}$-extensions, Proc. Japan Acad. Ser. A Math. Sci. 71 (1995), no. 9, 218-219.

[ 3 ] M. Ozaki, The class group of $\mathbf{Z}_{p}$-extensions over totally real number fields, Tohoku Math. J. (2) 49 (1997), no. 3, 431-435.

[ 4 ] L. C. Washington, Introduction to cyclotomic fields, 2nd ed., Graduate Texts in Mathematics, 83, Springer-Verlag, New York, 1997. 